University of Wollongong

Research Online

Faculty of Engineering and Information

Faculty of Engineering and Information

Sciences - Papers: Part B

Sciences

2020

Modeling and Motion Control of a Liquid Metal Droplet in a Fluidic Channel

Jie Xie

Fangxia Li

Shaolong Kuang

Hao Yang

Xiangpeng $\mathrm{Li}$

See next page for additional authors

Follow this and additional works at: https://ro.uow.edu.au/eispapers1

Part of the Engineering Commons, and the Science and Technology Studies Commons

Research Online is the open access institutional repository for the University of Wollongong. For further information contact the UOW Library: research-pubs@uow.edu.au 


\title{
Modeling and Motion Control of a Liquid Metal Droplet in a Fluidic Channel
}

\begin{abstract}
(C) 1996-2012 IEEE. As an emerging multifunctional material, Gallium-based room temperature liquid metal has attracted a lot of attention for a variety of applications due to its mobility and deformability. However, controlling the motion of a liquid metal droplet accurately still remains unrevealed, which restricts its application in many fields. In this article, we propose a hybrid framework that would control the motion of a liquid metal droplet in a one-dimensional (1-D) fluidic channel. A dynamic model of a liquid metal droplet immersed in the electrolyte when an electrical field is applied to each end of the channel is discussed first, followed by a setpoint controller designed to calculate the current input needed to drive the liquid metal droplet to its destination with vision feedback. To obtain the desired highresolution current output, a fast and high-resolution current output power supply will be established by integrating a fast PID controller and a simple programmable dc power supply. The effectiveness of this proposed approach will be verified by controlling a liquid metal droplet so that it reaches its destination inside the polymethyl methacrylate channel. In this article, the proposed approach may lead to the development of tiny soft robots, or microfluidic systems that can be driven accurately by the liquid metal droplets.
\end{abstract}

Disciplines

Engineering | Science and Technology Studies

Publication Details

Xie, J., Li, F., Kuang, S., Yang, H., Li, X., Tang, S., Li, W. \& Zhang, S. (2020). Modeling and Motion Control of a Liquid Metal Droplet in a Fluidic Channel. IEEE/ASME Transactions on Mechatronics, 25 (2), 942-950.

Authors

Jie Xie, Fangxia Li, Shaolong Kuang, Hao Yang, Xiangpeng Li, Shiyang Tang, Weihua Li, and Shiwu Zhang

This journal article is available at Research Online: https://ro.uow.edu.au/eispapers1/4016 


\title{
Modelling and Motion Control of a Liquid Metal Droplet in a Fluidic Channel
}

\author{
Jie Xie, Fangxia Li, Shaolong Kuang, Hao Yang, Xiangpeng Li, Shiyang Tang, Weihua Li, Shiwu \\ Zhang
}

\begin{abstract}
As an emerging multifunctional material, Gallium based room temperature liquid metal has attracted a lot of attention for a variety of applications due to its mobility and deformability. However, controlling the motion of a liquid metal droplet accurately still remains unrevealed, which restricts its application in many fields. In this paper we propose a hybrid framework that would control the motion of a liquid metal droplet in a one-dimensional fluidic channel. A dynamic model of a liquid metal droplet immersed in the electrolyte when an electrical field is applied to each end of the channel is discussed first, followed by a setpoint controller designed to calculate the current input needed to drive the liquid metal droplet to its destination with vision feedback. To obtain the desired high-resolution current output, a fast and high-resolution current output power supply will be established by integrating a fast PID controller and a simple programmable DC power supply. The effectiveness of this proposed approach will be verified by controlling a liquid metal droplet so that it reaches its destination inside the PMMA channel. This proposed approach may lead to the development of tiny soft robots, or micro-fluidic systems that can be driven accurately by liquid metal droplets.
\end{abstract}

Index Terms - Liquid metal droplets, dynamic model, setpoint control, surface tension, soft robotics

\section{INTRODUCTION}

$\mathrm{R}^{\mathrm{con}}$ OOM temperature liquid metal gallium and several of its alloys possess many remarkable properties such as high electrical and thermal conductivity, large surface tension (nearly ten times that of water), extremely low vapor pressure $\left(<10^{-6} \mathrm{~Pa}\right.$ at $\left.500^{\circ} \mathrm{C}\right)$, and low toxicity compared with mercury [1]. These properties have shown that room temperature liquid metals can be used for a wide range of applications such as soft and stretchable electronic components [2-5], micro-fluidics devices [6-8], small vehicles and robots $[9,10]$, soft sensors $[11,12]$, tunable antennas and apertures $[13,14]$, as well as fluidic optical components and displays $[15,16]$. Moreover, liquid metals have great potential for manufacturing the soft

This work is supported in part by grants from NSFC under Grant Nos. 51975550, 61873339, 61503270, 51828503, U1713206, a grant from National Science Foundation of Jiangsu Province under Grant No. BK20190096, a grant from China Postdoctoral Science Foundation under Grant No. 2016M590497, and supported by the State Key Laboratory of Applied Optics. Dr. Shi-Yang Tang is the recipient of the Vice-Chancellor's Postdoctoral Research Fellowship funded by the University of Wollongong, Australia. (Corresponding address: licool@ suda.edu.cn and swzhang@ustc.edu.cn)

J. Xie, and S. Zhang are with CAS Key Laboratory of Mechanical Behavior and Design of Materials, Department of Precision Machinery and Precision robots that are a big dream for mankind. However, controlling the surface tension of liquid metals to achieve the desired motion and deformation remains an open question that is the key to broaden their potential applications.

Many methods have already been developed to manipulate liquid metal droplets, all of which can be categorized as follows: electrical field [17, 18], magnetic field [19, 20], electrochemical reaction [21, 22], photochemistry [23] and ionic imbalance [24]. Among these methods, applying an external electrical field in ionic electrolyte to actively manipulate the surface tension of liquid metal droplets is the most popular because it is very convenient for practical purposes. According to Lippman's equation, the applied external electrical field can break the equilibrium of charge distribution of the electrical double layer (EDL) around the liquid metal-electrolyte interface and generate a surface tension gradient along the surface of liquid metal droplets that will drive their motion and deformation [17]. Several efforts relating to the issue of driving a single liquid metal droplet with an electrical field have already been investigated. For example, a DC electrical field was applied to induce a liquid metal mercury slug to move inside a microfluidics channel [25], and the average speed of mercury was estimated theoretically. Meanwhile, gallium based liquid metal droplets achieved linear and reciprocating motion under a DC and AC electrical field, respectively, as reported in $[17,26]$. More recently, a low power consumption and high efficiency micro-pump was introduced by applying an AC electrical field with DC offset to a Galinstan (68.5\% gallium, $21.5 \%$ indium, and $10 \%$ tin) droplet confined inside a cylindrical chamber embedded in fluidic chips [27]. Despite these achievements, accurately controlling the motion of a liquid metal droplet still remains unrevealed.

In this study we have developed a hybrid control framework which combines a setpoint controller and a fast PID controller to control the motion of a liquid metal droplet in a onedimensional channel filled with alkali electrolyte by using a simple programmable DC power supply. To achieve this

Instrumentation, University of Science and Technology of China, Hefei, Anhui 230027, China (e-mail: swzhang@ustc.edu.cn).

F. Li, S. Kuang, H. Yang, and X. Li are with Robotics and Microsystems Center, Collage of Mechanical and Electrical Engineering, Soochow University, China. X. Li is also with the State Key Laboratory of applied optics, China (e-mail: licool@ suda.edu.cn).

S. Tang and W. Li are with School of Mechanical, Materials Mechatronic and Biomedical Engineering, University of Wollongong, Australia (e-mail: shiyang@uow.edu.au ). 
objective, a dynamic model of the liquid metal droplet immersed in a fluidic channel filled with alkali electrolyte was established by analyzing related forces including the surface tension induced driving force, the viscous drag force, and the friction force between the liquid metal and the channel. The setpoint controller was designed to calculate the desired current input to drive the liquid metal droplet to its destination with vision feedback. To obtain the desired high-resolution current output, a fast and high-resolution current output power supply was established by integrating the PID controller and a simple programmable DC power supply. Experiments on controlling the Galinstan droplets to their destinations inside a PMMA fluidic channel demonstrated the effectiveness and the accuracy of this proposed method. To the best of our knowledge, this is the first investigation into controlling the motion of liquid metal droplets in fluidic channels. This proposed method may promote the development of new liquid metal enabled robots.

The rest of this paper is organized as follows. Section II introduces the liquid metal manipulation system we established. The dynamic model of the liquid metal droplet is given in Section III. In Section IV, a setpoint controller is proposed to control the liquid metal droplet inside the channel. Experiments are performed to validate the proposed method in Section V, and the conclusion is presented in Section VI.

\section{THE SYSTEM FOR MANIPULATING LIQUID METAL DROPLETS}

To accurately control the motion of liquid metal droplets, the driving mechanism must be obtained and the manipulation system established.

\section{A. Preliminary}

When a gallium-based liquid metal droplet makes contact with the alkali electrolyte, the gallium inside the Galinstan can slowly react with the alkali electrolyte and produces gallates $\left[\mathrm{Ga}(\mathrm{OH})_{4}\right]^{-}$at the liquid metal-electrolyte interface [17]. This concentration of gallates $\left[\mathrm{Ga}(\mathrm{OH})_{4}\right]^{-}$makes the surface of the liquid metal droplet negatively charged and leads to an accumulation of positively charged ions in the diffuse layer of EDL. By modelling the EDL as an idealized Helmgoltz-Perrin model [28], the relationship between the surface tension and the potential difference across the EDL can be formulated by Lippman's equation and expressed as follows

$$
\gamma(l)=\gamma_{0}-\frac{1}{2} c_{p}(l) \cdot V^{2}(l)
$$

where $l \in S_{l}$ denotes a single point on the surface of the droplet, $S_{l}$ denotes the whole surface of the droplet, $\gamma(l)$ denotes the surface tension of the droplet in the neighborhood of point $l, c_{p}(l)$ is the capacitance per unit area of the EDL in the neighborhood of point $l, \gamma_{0}$ denotes the maximum surface tension when $V(l)=0$, and $V(l)$ is the potential difference across the liquid metal-electrolyte interface in the neighborhood of point $l$ and can be formulated as follows

$$
V(l)=\varphi_{e}(l)-\varphi_{m}(l)
$$

where $\varphi_{e}(l)$ denotes the electrical potential of the EDL at the side of the electrolyte solution, and $\varphi_{m}(l)$ denotes the electrical potential of the EDL at the side of the surface of liquid metal. Note that the EDL electrically isolates the liquid metal droplet from the electrolyte, so the liquid metal droplet in the electrolyte behaves as if it is a nonconductor and the surface of the droplet is potential equivalent [25], thus $\varphi_{m}(l)$ can be treated as a constant $\varphi_{l}$, which can be written as $\varphi_{m}(l)=\varphi_{l}$.

The potential is approximately uniform throughout the droplet so the ionic distribution in the EDL is also uniform, as shown in Fig. 1(a). When an external voltage is applied, there will be a potential gradient along the EDL which will help to redistribute the ionic in the EDL and thus induce surface charge redistribution similar to that shown in Fig. 1(b). The surfaces facing the anode accumulate more charges than the surfaces facing the cathode. The surface charge redistribution leads to the formation of a surface tension gradient at the liquid metalelectrolyte interface and results in a pressure difference gradient at the interface which can be formulated by Young-Laplace's equation as follows [29]:

$$
P(l)=\gamma(l) \cdot\left(\frac{1}{R_{1}(l)}+\frac{1}{R_{2}(l)}\right)
$$

where $P(l)$ denotes the pressure difference in a small neighborhood of point $l$ on the surface of a liquid metal droplet, which is normal to the neighborhood and points to the interior of the liquid metal droplet [29]. $R_{1}(l)$ and $R_{2}(l)$ denote the two principal curvature radii of the neighborhood of point $l$ respectively, as shown in Fig. 1(c). The total pressure difference gradient between the surface of the liquid metal and the electrolyte can produce a driving force that actuates the

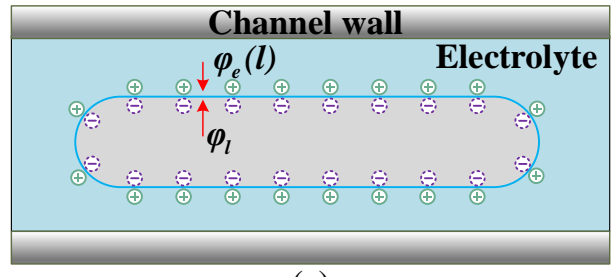

(a)

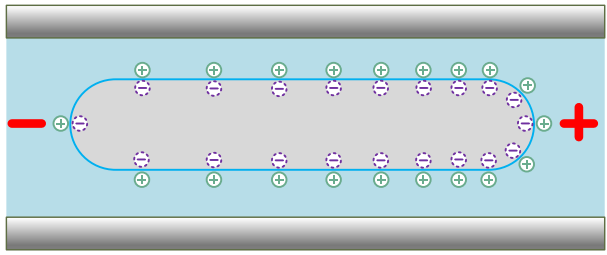

(b)

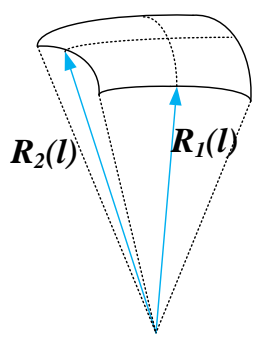

(c)
Fig. 1 (a) EDL of a liquid metal droplet with initial charge; (b) Surface charge redistribution under external potential field; (c) Principal curvature radii of the neighborhood of point $l$. 


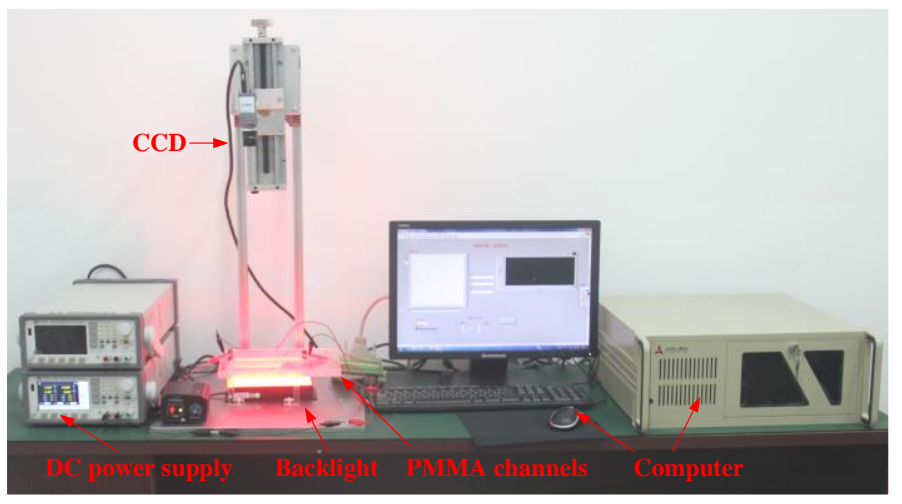

(a)

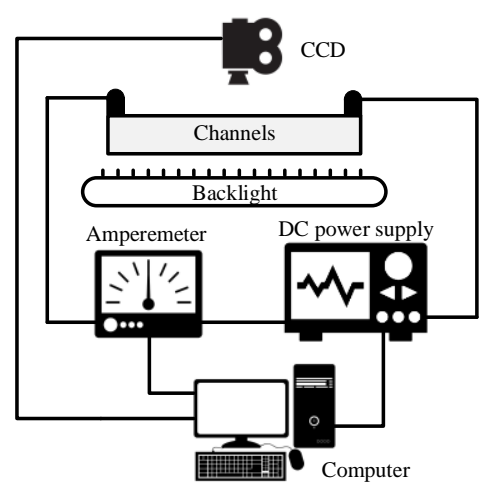

(b)

Fig. 2 (a) The photo of the liquid metal manipulation system; (b) The hybrid control framework of the liquid metal manipulation system.

liquid metal droplet to flow in the direction of low surface tension.

\section{B. System setup}

To control the movement of a liquid metal droplet accurately with an external electrical field, we established the manipulation system shown in Fig. 2(a). This system has three modules for executive, sensory, and control, as shown in Fig. 2(b). The executive module has a fluidic chip with two opentop polymethylmethacrylate (PMMA) channels with circular and rectangular cross sections. All of these channels were filled with $\mathrm{NaOH}(0.5 \mathrm{~mol} / \mathrm{L})$ solution as the electrolyte. Liquid metal droplets were positioned in the channels and immersed inside the $\mathrm{NaOH}$ solutions. The volume of liquid metal droplets was governed by a numerical injection pump (LSP02-1B, Longer, the UK). Graphite electrodes were mounted at two ends of each channel to input the external electrical field generated by a programmable DC power supply (IT6432, ITECH, USA). The sensory modular contains a current measurement module within the DC power supply to measure the current input to the control circuit and an IEEE 1394 CCD camera (XCD-SX90, Sony, Japan) to capture the position of the liquid metal droplets. An LED backlight was placed below the channel to provide reliable lighting conditions for the vision system. The control module used in the liquid metal droplet manipulation system was developed with Labview and run in an industrial computer (RK610IMB-M42H, ADLINK, China).

\section{DYNAMIC MODEL}

The problem we investigated is formulated as follows: In a liquid metal droplet manipulation system, determine a method to control the current input $i$, such that the liquid metal droplet can be driven automatically and precisely to any desired position $g_{q}$ in the fluidic channel. Therefore to control the motion of a liquid metal droplet we must first derive a dynamic model of the droplet.

\section{A. Dynamics}

When a liquid metal droplet is actuated by an external electrical field to move in channel, the main forces that govern its motion are the surface tension induced driving force $F_{t}$, the

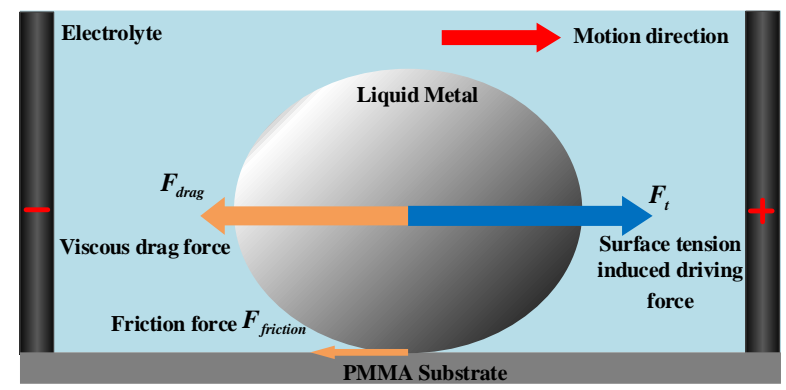

Fig. 3 The force analysis of a liquid metal droplet in electrical field.

viscous drag force $F_{d r a g}$ caused by the electrolyte, and the friction force $F_{\text {friction }}$ between the liquid metal and the channel, as shown in Fig.3. The dynamics of the liquid metal droplet are then given by

$$
F_{t}-F_{\text {drag }}-F_{\text {friction }}=m \ddot{q}
$$

where $m$ denotes the mass of the liquid metal droplet, and $q$ denotes its position.

The viscous drag force $F_{d r a g}$ can be expressed as follows [30]:

$$
F_{d r a g}=2 \pi r v \frac{3 \mu_{e}+2 \mu_{l}}{\mu_{e}+\mu_{l}}
$$

where $r$ denotes the radius of the liquid metal droplet, $v$ is its translational speed, $\mu_{e}$ denotes the viscosity of the electrolyte (about $1.4 \times 10^{-3} \mathrm{~Pa} \cdot s$ at $20^{\circ} \mathrm{C}$ ) and $\mu_{l}$ denotes the viscosity of the liquid metal (about $2.4 \times 10^{-3} \mathrm{~Pa} \cdot s$ at $20^{\circ} \mathrm{C}$ [7]).

The friction force $F_{\text {friction }}$ is formulated as follows

$$
F_{\text {friction }}=\mu_{f} \rho_{\text {diff }} \theta g
$$

where $\mu_{f}$ denotes the coefficient of friction between the liquid metal droplet and the fluidic channel, $\rho_{\text {diff }}$ is the difference in density between the droplet and the electrolyte, $\theta$ represents 
the volume of the droplet, and $g$ denotes gravitational acceleration.

\section{B. Surface Tension Induced Driving Force}

The surface tension induced driving force $F_{t}$ of the whole liquid metal droplet can then be formulated as follows:

$$
\vec{F}_{t}=\int_{l \in S_{l}} P(l) \cdot \vec{l}_{n} \cdot d s(l)
$$

where $s(l)$ denotes the neighborhood area of point $l$, and $\vec{l}_{n}$ denotes the normal vector of the neighborhood of point $l$.

By substituting (1) and (3) into (7), the surface tension induced driving force $F_{t}$ can be rewritten as

$$
\vec{F}_{t}=\int_{l \in S_{l}}\left(\gamma_{0}-\frac{c_{p}(l)}{2} V(l)^{2}\right)\left(\frac{1}{R_{1}(l)}+\frac{1}{R_{2}(l)}\right) \cdot \vec{l}_{n} \cdot d s(l)
$$

Note that the potential difference across the EDL $V(l)$ is difficult to obtain under different external voltages, which makes the exact calculation of the surface tension induced driving force $F_{t}$ difficult. In the following development, we will address this challenge and obtain the surface tension induced driving force $F_{t}$.

Firstly, the charges of EDL $Q$ can be formulated as follows

$$
Q=\int_{l \in S_{l}} d Q(l)=\int_{l \in S_{l}} V(l) \cdot d c(l)
$$

where $Q(l)$ denotes the charge distribution function of the EDL in the neighborhood of point $l ; c(l)=k s(l)$ is the capacitance of the EDL in the neighborhood of point $l, k=\frac{\varepsilon \varepsilon_{0}}{d_{0}}$ is a capacitance related parameter, where $\varepsilon$ denotes the relative permittivity, $\varepsilon_{0}$ is the vacuum permittivity, and $d_{0}$ is the thickness of the EDL.

The electrical potential of the EDL at the electrolyte side is potential equivalent and the charges of the EDL are uniformly distributed along the surface of liquid metal droplet with no externally applied electrical field. The total charges of the EDL termed initial charges of EDL $Q_{0}$ is formulated as follows

$$
Q_{0}=V_{0} C_{0}
$$

where $V_{0}$ denotes the potential difference across the EDL with no external applied electrical field, $C_{0}$ denotes the total capacitance of the EDL with no externally applied electrical field.

Assumption 1: The total charges and capacitance of the EDL are conserved under external electrical fields, i.e. $Q \equiv Q_{0}$ and
$C_{0} \equiv \int_{l \in S_{l}} d c(l)$.

Based on Assumption 1, the following Theorem 1 can be obtained.

Theorem 1: When an external potential is applied there is always at least a point $l_{p}=\left\{l \mid \varphi_{e}\left(l_{p}\right)=\bar{\varphi}_{e}(l)\right\}$ on the surface of the liquid metal, which makes $V\left(l_{p}\right)=V_{0}$, where $\bar{\varphi}_{e}(l)$ denotes the mean value of $\varphi_{e}(l)$.

Proof: See Appendix A.

Remark 1: Theorem 1 bridges the gap between the potential difference of the EDL $V(l)$ and the electrical potential of the EDL at the electrolyte side $\varphi_{e}(l)$, which is easy to model and calculate. That is the key to modeling and calculating the surface tension induced driving force $F_{t}$.

Based on Theorem 1, (2) can be written as:

$$
V(l)=\varphi_{e}(l)-\varphi_{l}=\varphi_{e}(l)-\left(\varphi_{e}\left(l_{p}\right)-V_{0}\right)=\left(\varphi_{e}(l)-\bar{\varphi}_{e}(l)\right)+V_{0}
$$

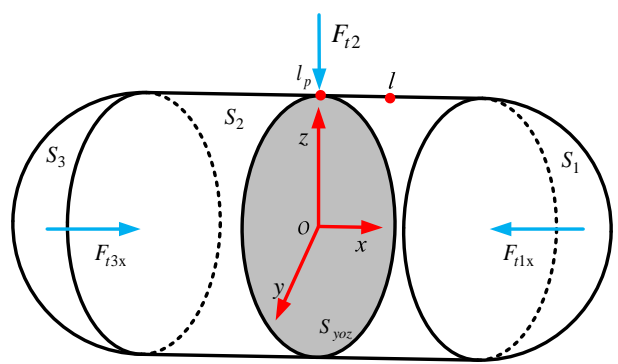

Fig. 4 Appearance of the liquid metal droplet.

We then calculated the surface tension induced driving force $F_{t}$ for a liquid metal droplet moving in a one dimensional channel. This process includes two steps as follows:

Step 1: Find $l_{p}$ : a liquid metal droplet looks like a worm when it moves in a straight channel, as shown in Fig.4. The middle is a cylinder and each end has irregular surfaces which are symmetrical in the direction of the channel. A local coordinate is fixed in the geometric center of the liquid metal droplet, where $o$ denotes the origin, $x$ denotes the axis along the channel, and $y$ and $z$ denote the other two coordinate axes. Due to the highly symmetrical geometrical shape of the liquid metal droplet, $l_{p} \in\left\{l \mid S_{y o z} \cap S_{l}\right\}$, where $S_{y o z}$ is a plane passing the $y$ and $z$ axis and perpendicular to $x$-axis, as shown in Fig. 4. In a one dimensional channel, only the forces along the channel are considered, so for any point $l \in S_{l}$, we have

$$
\varphi_{e}(l)=\varphi_{e}\left(l_{p}\right)+I \Omega(l)=\bar{\varphi}_{e}(l)+I \Omega(l)
$$

where the straight line passing the two points $l_{p}$ and $l$ is parallel to the $x$-axis, $I$ denotes the current of the electrolyte, $\Omega(l)$ denotes the resistance distribution function along the $x$ axis from $l_{p}$ to $l$, which can be evaluated by the thin layer of 
electrolyte between the liquid metal droplet and channel wall. Due to the highly symmetrical geometrical shape of the liquid metal droplet, $\Omega(l)$ is an odd function with respect to plane $S_{y o z}$.

Step 2: Calculate $F_{t}$ : following Step 1, using (11) and (12), (8) can be simplified as

$$
\vec{F}_{t}=\int_{l \in S_{l}}\left(\gamma_{0}-\frac{c_{p}(l)\left(I \Omega(l)+V_{0}\right)^{2}}{2}\right)\left(\frac{1}{R_{1}(l)}+\frac{1}{R_{2}(l)}\right) \cdot \vec{l}_{n} \cdot d s(l)
$$

\section{Calculation of the Driving Force}

In our experiment the appearance of the liquid metal droplet in the channel is shown in Fig. 4. We assumed that the anode is in the direction of the $+x$-axis and the cathode is in the direction of the $-x$-axis. We also assumed $c_{p}(l)=c\left(l \in S_{l}\right)$, where $c$ is the capacitance per unit area of the EDL. Since $\varphi_{e}(l)$ is the same on the plane which is perpendicular to the $x$-axis and parallel to $S_{y o z}, \Omega(l)$ is a function only related to the $x$ axis, so it can be expressed as $\Omega(l)=\Omega\left(l_{x}\right)$, where $l_{x}$ is the $x$ position of the point $l$. We divided the liquid metal droplet into three surfaces to calculate the driving force caused by the pressure difference across the EDL respectively; they are the right surface $S_{1}=S(x, y, z)$, the cylindrical surface $S_{2}$ and the left surface $S_{3}=S(-x, y, z)$, where $S(x, y, z)$ is a function of the surface $S_{1}$. The driving force induced by $S_{1}, S_{2}$ and $S_{3}$ are $F_{t 1}, F_{t 2}$ and $F_{t 3}$ respectively. The direction of $F_{t 2}$ is perpendicular to the channel, so $F_{t 2}$ does not contribute to the movement. We only need to consider the driving force along the channel, that is, $F_{t 1 \mathrm{x}}$ and $F_{t 3 \mathrm{x}}$, where $F_{t 1 \mathrm{x}}$ and $F_{t 3 \mathrm{x}}$ denote the component of the driving force $F_{t 1}$ and $F_{t 3}$ on the $x$-axis, respectively. Using (13), the driving force $F_{t}$ can be obtained as

$$
F_{t}=2 q_{0} I \int_{l \in S(x, y, z)} \Omega\left(l_{x}\right)\left(\frac{1}{R_{1}(l)}+\frac{1}{R_{2}(l)}\right) d y d z
$$

where $q_{0}$ is the total charges per unit area of the EDL (the typical value of $q_{0}$ is $0.05 \mathrm{C} / \mathrm{m}^{2}$ [27]). The calculation process can be seen in Appendix B.

\section{Control Design}

A setpoint controller was used to control the motion of a liquid metal droplet as inspired by [31, 32]. The dynamics of the liquid metal droplet are given by:

$$
m \ddot{q}+K_{2} \dot{q}+K_{3}=K_{1} I=i
$$

where $\quad K_{1}=2 q_{0} \int_{l \in S(x, y, z)} \Omega\left(l_{x}\right)\left(\frac{1}{R_{1}(l)}+\frac{1}{R_{2}(l)}\right) d y d z$, $K_{2}=2 \pi r \frac{3 \mu_{e}+2 \mu_{l}}{\mu_{e}+\mu_{l}}, K_{3}=\mu_{f} \rho_{\text {diff }} \theta g$, which are all positive constants.

A setpoint controller used to drive a liquid metal droplet to the desired position in a straight channel was formulated as follows:

$$
i=-K_{p} \tanh \left(K_{r}\left(q-g_{q}\right)\right)-K_{q} \dot{q}+K_{3}
$$

where $K_{p}$ and $K_{r}$ are the positive control gains, $K_{q}$ is the control gain which satisfies the condition of $K_{q}>-K_{2}$. The hyperbolic tangent function was used to bound the term $K_{p} K_{r}\left(q-g_{q}\right)$ within a certain range $\left(-K_{p}, K_{p}\right)$ and avoid generating a large control input $i$ in case the liquid metal droplet is located a long way from the desired $g_{q}$. When the input current is too large, serious electrolysis may appear at the interface between the electrode and the electrolyte. Gas bubbles generated in the electrolysis reaction may escape into the electrolyte solution and hamper the motion of liquid metal. We also noticed some slight electrolysis at the interface between the surface of the liquid metal droplet and the electrolyte when a large current was applied; this also had minor effects on the motion of liquid metal [33]. By substituting the controller (16) into the dynamics (15), the closed-loop dynamics is as

$$
m \ddot{q}+\left(K_{2}+K_{q}\right) \dot{q}+K_{p} \tanh \left(K_{r}\left(q-g_{q}\right)\right)=0
$$

Theorem 2: The liquid metal manipulation system (4) is globally asymptotically stable under the controller (16).

Proof: Consider a Lyapunov function $V \geq 0$ as follows

$$
V=\frac{1}{2} m \dot{q}^{2}+\frac{K_{p}}{K_{r}} \ln \left(\cosh \left(K_{r}\left(q-g_{q}\right)\right)\right)
$$

Taking the derivation of $V$ with respect to time yields

$$
\dot{V}=m \dot{q} \ddot{q}+K_{p} \tanh \left(K_{r}\left(q-g_{q}\right)\right) \dot{q}
$$

By substituting (17) into (19), and considering the condition where $K_{q}>-K_{2}$, we have

$$
\dot{V}=-\left(K_{2}+K_{q}\right) \dot{q}^{2} \leq 0
$$

The final calculation shows that the liquid metal manipulation system (4) is globally asymptotically stable under the controller (16). 
Theorem 3: Considering the liquid metal manipulation system (4), the proposed setpoint controller (16) gives rise to the convergence of $q \rightarrow \mathrm{g}_{q}$ and $\dot{q} \rightarrow 0$.

Proof: Since $\dot{V}=0$ implies $\dot{q}=0$ as $t \rightarrow \infty$, the maximum invariant set is $K_{p} \tanh \left(K_{r}\left(q-g_{q}\right)\right)=0$. Hence, $q=g_{q}$ and $\dot{q}=0$.

\section{EXPERIMENTS}

This section describes the experimental setup and the results based on the proposed controller.

\section{A. Experimental Setup}

In the experiment, the middle part of the liquid metal is a cylinder and the ends are hemispheres. The length of the cylinder is $L$ and the radii of the two hemispheres are $R$, which means $R_{1}(l)=R_{2}(l)=R . \Omega(l)$ is a linear function along the $x$-axis, which can be expressed as $\Omega(l)=\frac{R_{L}}{L+2 R} l_{x}$, where $R_{L}$ is the total resistance of the thin layer of electrolyte between the liquid metal droplet and the channel wall. $S(x, y, z)$ can be expressed as:

$$
S(x, y, z)=\left(x-\frac{L}{2}\right)^{2}+y^{2}+z^{2}=R^{2}\left(x \geq \frac{L}{2}\right)
$$

So $K_{1}$ can be calculated as:

$$
K_{1}=2 \pi q_{0} R R_{L} \frac{L+\frac{4}{3} R}{L+2 R}
$$

In the experiment the volume of the liquid metal droplet was $0.1 \mathrm{ml}$ and its shape in the channel was calibrated dynamically by the CCD camera with $1280 \times 960$ pixels. Each pixel was calibrated as $0.25 \mathrm{~mm}$. For a liquid metal droplet of $0.1 \mathrm{ml}$, its radius was almost $0.003 \mathrm{~m}$. From the definition in (15), we obtain that $K_{2}=0.045$ and $K_{3}=0.0001$. The configurations of the open-top PMMA channels used in the experiment are listed in Table 1. The cross sections of the channels are shown in Fig. 5. The height of the solution must enable the solution to cover the liquid metal.

The liquid metal droplet is very sensitive to the circuit current due to its very high surface tension, so a small current drift of 2 $\mathrm{mA}$ in the circuit was enough to induce a $0.1 \mathrm{ml}$ static liquid metal droplet to move. The accuracy of the current output module of the DC power supply was more than $2 m A$ and could not provide enough current accuracy so we proposed a current output strategy that would impart a high resolution current output to the circuit, as shown in Fig. 6. The voltage output module of the DC power supply with an accuracy of $5 \mathrm{mV}$ was used for the output voltages to the two electrodes. The current measurement module of the DC power supply with measurement accuracy of $5 u \mathrm{~A}$ was used to measure the current input to the control circuit and also take any feedback to a PID controller formulated as follows:

$$
\Delta U=k_{i} e_{k}+k_{d}\left(e_{k}-2 e_{k-1}+e_{k-2}\right)+k_{p}\left(e_{k}-e_{k-1}\right)
$$

where $e_{k}, e_{k-1}, e_{k-2}$ are the current errors of the latest three measurements; $\Delta U$ denotes the output voltage error; $k_{i}=215$, $k_{d}=30$, and $k_{p}=56.9$ are three control gains respectively.

Thanks to the above strategy, the current output accuracy of the augmented power supply was less than $0.1 \mathrm{~mA}$, the current output resolution reached $0.01 \mathrm{~mA}$, and the response time was less than $200 \mathrm{~ms}$.

\section{B. Results of the Experiments}

In the experiment the liquid metal droplets were driven from pre-set initial positions to different destinations and travelled different distances in channels with circular and rectangular cross sections to evaluate the performance of the proposed control method. We repeated the experiments more than 200 times and the droplet arrived at its destination successfully every time.

Figure 7 shows the manipulation process taken from a video. The manipulation task shown in the five figures on the left (a)(e) took place in a circular channel while the five figures on the right (f)-(j) took place in a rectangular channel. The liquid metal is shown as a disc and its destination is denoted by a
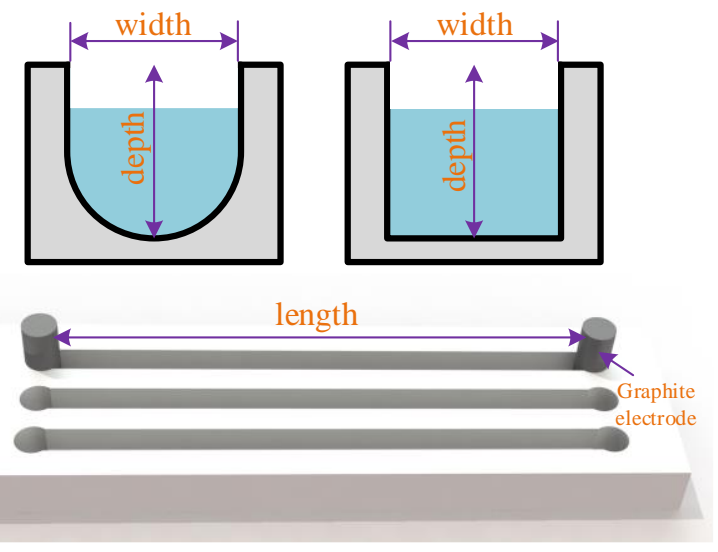

Fig. 5 Channels used in the experiments.

Table1 The configuration of the channels used in the experiments.

\begin{tabular}{ccccc}
\hline \hline Index & $\begin{array}{c}\text { Cross } \\
\text { section }\end{array}$ & $\begin{array}{c}\text { Width } \\
(\mathrm{mm})\end{array}$ & $\begin{array}{c}\text { Depth } \\
(\mathrm{mm})\end{array}$ & $\begin{array}{c}\text { Length } \\
(\mathrm{mm})\end{array}$ \\
\hline 1 & circular & 8 & 8 & 150 \\
\hline 2 & rectangular & 8 & 8 & 150 \\
\hline \hline
\end{tabular}

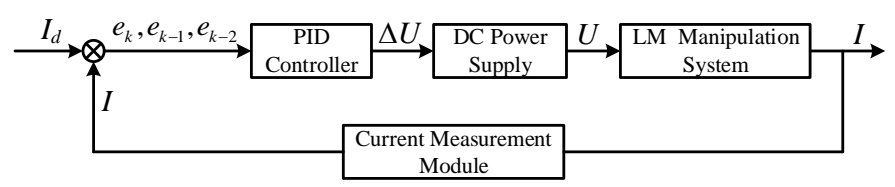

Fig. 6 Schematic diagram of the high resolution current output strategy. 


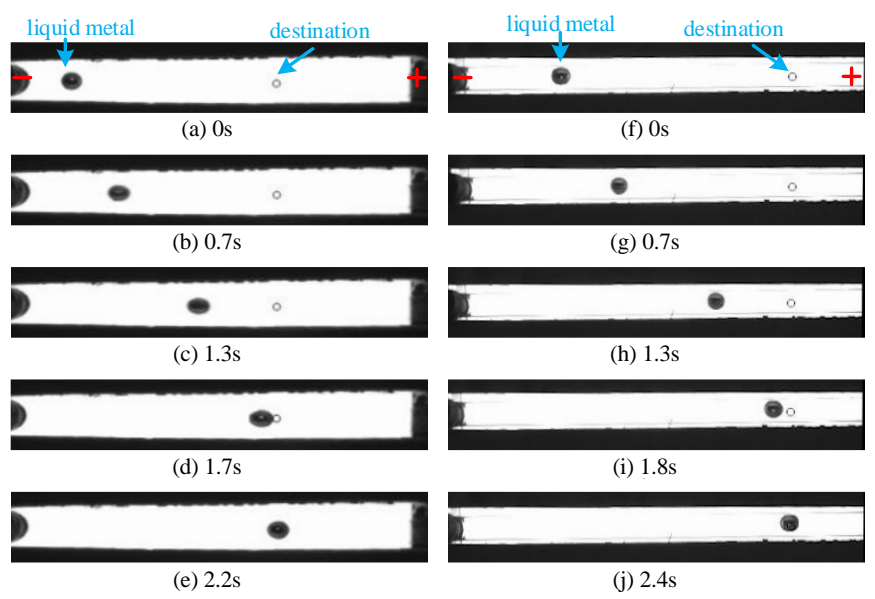

Fig. 7 Liquid metal droplet manipulation process. (a)-(e) Circular channel. (f)(j) Rectangular channel.
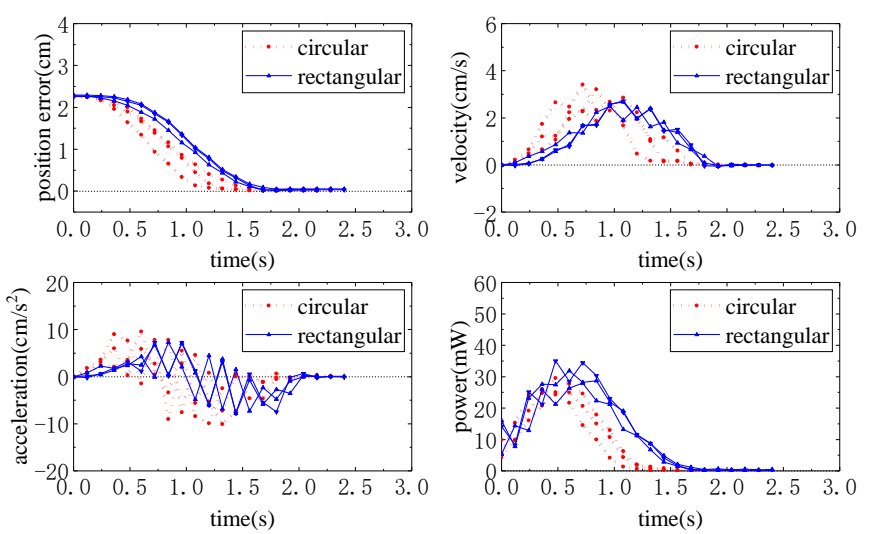

Fig. 8 The control performance comparison when travelling short distance.
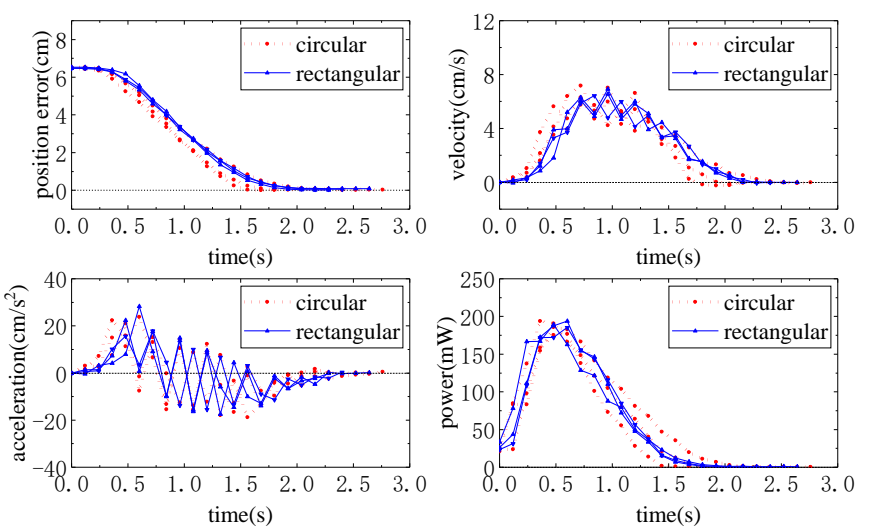

Fig. 9 The control performance comparison when travelling long distance.
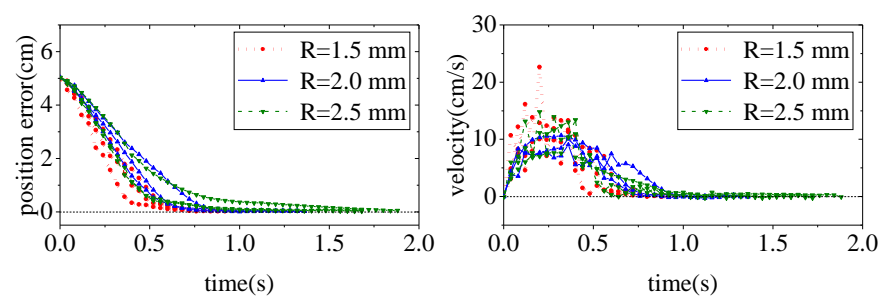

Fig. 10 The control performance comparison of droplets with different radii when travelling in circular channel.

small circle. Fig. 7(a) and Fig. 7(f) show the initial configuration, whereas Figs. 7(b)-(d) and Figs. 7(g)-(i) show the manipulation process at different time. Fig. 7(e) and Fig. 7(j) show that the liquid metal droplet successfully arrived at its destination. The supplementary movie can be seen in Movie S1 (circular channel) and S2 (rectangular channel).

The control performance was compared by driving liquid metal droplets travelling long $(65 \mathrm{~mm})$ and short distances $(23 \mathrm{~mm})$ in different channels under the proposed control methods shown in Fig. 8 and Fig. 9, respectively. In each figure, we randomly selected 6 results from two channels for further comparison. The curves with a red dashed line denote the results in circular channels and the curves with solid blue line denote the results from rectangular channels. Note that the position errors, the velocities and accelerations all converge to zero in every experiment, which implies that all the liquid metals were successfully driven to their destinations. The average position errors for travelling short distances and long distances in a circular channel are $0.026 \mathrm{~cm}$ and $0.055 \mathrm{~cm}$, and $0.037 \mathrm{~cm}$ and $0,060 \mathrm{~cm}$ for travelling short distances and long distances in a rectangular channel. The proposed control method was valid for different types of channels. Furthermore, the speed at which the liquid metals converged in the circular channel was slightly faster than in the rectangular channel, and less power was consumed in the circular channel. This result was due to the thin layer of electrolyte between the liquid metal and the circular channel was even narrow that of the rectangular channel, which made it easier for the liquid metal droplet to obtain a larger surface tension induced driving force according to (14). Finally, the maximal velocity of the liquid metal reached $7.5 \mathrm{~cm} / \mathrm{s}$ (almost 12.5 its body length per second), and the total power consumption was less than $200 \mathrm{~mW}$. This result implies that the proposed control method has a high driving efficiency.

In our experiments the size of the liquid metal had almost no effect on the control performance. We can control millimeterscale liquid metal with variable sizes (See Movie S3) and the final position errors are almost the same for different sizes, as shown in Fig. 10. In our experiments the smallest radius of the liquid metal droplet that can be driven was 500 microns.

\section{CONCLUSION}

This paper proposed a hybrid control framework that would drive a liquid metal droplet to a desired position in a one dimensional fluidic channel. To achieve this objective, the dynamic model of a liquid metal droplet immersed in fluidic channels with an external electrical field was investigated. A setpoint controller was then designed to drive the liquid metal droplet to its destination with the help of vision feedback and a high-resolution current generator achieved by integrating a high resolution current output strategy into a simple programmable DC power supply. Furthermore, serial experiments were carried out to demonstrate the effectiveness of the proposed control method. This controller can be utilized in many pragmatic fields such as microfluidics, miniaturized vehicles, flexible microrobots, and so on. We believe that this study could advance the 
development of smart, biomimetic and soft robots in the coming future.

\section{APPENDIX}

\section{A. Proof of Theorem 1}

When an external potential is applied, the charges of the EDL may be redistributed. Charges at the surface facing the cathode are attracted to the surface facing the anode. Since $Q(l)$ is continuous and $Q(l)=V(l) c(l)$, the potential difference function $V(l)$ is also continuous. Based on assumption 1, if the total charges $Q_{0}$ are conserved and $V(l)$ is continuous, we have

$$
M=\min V(l) \leq V_{0} \leq \max V(l)=N
$$

where $M$ and $N$ are constants. According to the intermediate value theorem [34], there may exist at least a point $l_{p} \in S_{l}$ that makes $V\left(l_{p}\right)=V_{0}$.

Based on Assumption 1, the total charges and capacitance of the EDL are conserved. Thus, we have

$$
Q_{0}=\int_{l \in S_{l}} V(l) d c(l)=V_{0} C_{0}
$$

Furthermore,

$$
\begin{aligned}
V_{0} & =\frac{1}{C_{0}} \int_{l \in S_{l}} V(l) d c(l) \\
& =\frac{1}{C_{0}} \int_{l \in S_{l}}\left(\varphi_{e}(l)-\varphi_{l}\right) d c(l) \\
& =\frac{1}{C_{0}} \int_{l \in S_{l}} \varphi_{e}(l) d c(l)-\frac{1}{C_{0}} \int_{l \in S_{l}} \varphi_{l} d c(l) \\
& =\frac{1}{C_{0}} \int_{l \in S_{l}} \varphi_{e}(l) d c(l)-\varphi_{l}
\end{aligned}
$$

According to the mean value theorem for double integrals [35], define $\bar{\varphi}_{e}(l)=\frac{1}{C_{0}} \int_{l \in S_{l}} \varphi_{e}(l) d c(l),(26)$ can be rewritten as

$$
V_{0}=\bar{\varphi}_{e}(l)-\varphi_{l}
$$

Based on the aforementioned proof, there may exist at least a point $l_{p} \in S_{l}$ that makes $V\left(l_{p}\right)=V_{0}$, so we also have

$$
V\left(l_{p}\right)=V_{0}=\varphi_{e}\left(l_{p}\right)-\varphi_{l}=\bar{\varphi}_{e}(l)-\varphi_{l}
$$

It is finally concluded that $\varphi_{e}\left(l_{p}\right)=\bar{\varphi}_{e}(l)$.

\section{B. Calculation of the Driving Force}

Using (13), $F_{t 1 \mathrm{x}}$ and $F_{t 3 \mathrm{x}}$ can be expressed as:

$$
\begin{aligned}
& F_{t 1 \mathrm{x}}=\int_{l \in S(x, y, z)}\left(\gamma_{0}-\frac{c}{2}\left(I \Omega\left(l_{x}\right)+V_{0}\right)^{2}\right)\left(\frac{1}{R_{1}(l)}+\frac{1}{R_{2}(l)}\right) \cdot \cos \left(\vec{l}_{n}, x\right) d s(l) \\
& =-\int_{l \in S(x, y, z)}\left(\gamma_{0}-\frac{c}{2}\left(I \Omega\left(l_{x}\right)+V_{0}\right)^{2}\right)\left(\frac{1}{R_{1}(l)}+\frac{1}{R_{2}(l)}\right) d y d z
\end{aligned}
$$

$$
\begin{aligned}
& F_{t 3 \mathrm{x}}=\int_{l \in S(-x, y, z)}\left(\gamma_{0}-\frac{c}{2}\left(I \Omega\left(l_{x}\right)+V_{0}\right)^{2}\right)\left(\frac{1}{R_{1}(l)}+\frac{1}{R_{2}(l)}\right) \cdot \cos \left(\vec{l}_{n}, x\right) d s(l) \\
& =\int_{l \in S(-x, y, z)}\left(\gamma_{0}-\frac{c}{2}\left(I \Omega\left(l_{x}\right)+V_{0}\right)^{2}\right)\left(\frac{1}{R_{1}(l)}+\frac{1}{R_{2}(l)}\right) d y d z \\
& =\int_{l \in S(x, y, z)}\left(\gamma_{0}-\frac{c}{2}\left(I \Omega\left(l_{-x}\right)+V_{0}\right)^{2}\right)\left(\frac{1}{R_{1}(l)}+\frac{1}{R_{2}(l)}\right) d y d z
\end{aligned}
$$

where $\left(\vec{l}_{n}, x\right)$ is the angle between $\vec{l}_{n}$ and $x$-axis. Since $\Omega(l)$ is an odd function with respect to the plane $S_{y o z}$, we have $\Omega\left(l_{-x}\right)=-\Omega\left(l_{x}\right)$, and the driving force $F_{t}$ can be obtained as

$$
\begin{aligned}
& F_{t}=F_{t 1 x}+F_{t 3 x} \\
& =2 c V_{0} I \int_{l \in S(x, y, z)} \Omega\left(l_{x}\right)\left(\frac{1}{R_{1}(l)}+\frac{1}{R_{2}(l)}\right) d y d z
\end{aligned}
$$

Since $q_{0}=c V_{0}[36]$, (31) can be expressed as:

$$
F_{t}=2 q_{0} I \int_{l \in S(x, y, z)} \Omega\left(l_{x}\right)\left(\frac{1}{R_{1}(l)}+\frac{1}{R_{2}(l)}\right) d y d z
$$

\section{REFERENCE}

[1] T. Y. Liu, P. Sen, and C. J. Kim, "Characterization of nontoxic liquidmetal alloy galinstan for applications in microdevices," $J$. Microelectromech. Syst., vol. 21, no. 2, pp. 443-450, Apr, 2012.

[2] J. A. Rogers, T. Someya, and Y. Huang, "Materials and mechanics for stretchable electronics," Science, vol. 327, no. 5973, pp. 1603-1607, Mar. 2010.

[3] E. Ozbay, "Plasmonics: merging photonics and electronics at nanoscale dimensions," Science, vol.311, no. 5758, pp. 189-193, Jan. 2006.

[4] C. Monat, P. Domachuk, and B. J. Eggleton, "Integrated optofluidics: a new river of light," Nat. Photonics, vol.1, no. 2, pp. 106-114, Feb. 2007.

[5] D. Schurig et al, "Metamaterial electromagnetic cloak at microwave frequencies," Science, vol. 314, no. 5801, pp. 977-980, Nov. 2006.

[6] M. G. Mohammed and M. D. Dickey, "Strain-controlled diffraction of light from stretchable liquid metal micro-components," Sens. Actuator APhys., vol. 193, no.4, pp. 246-250, Apr. 2013.

[7] K. Khoshmanesh et al, "Liquid metal enabled microfluidics," Lab Chip, vol. 17, no. 6, pp. 974-993, Feb. 2017.

[8] A. C. Siegel et al, "Cofabrication: a strategy for building multicomponent microsystems," Accounts Chem. Res., vol. 43, no. 4, pp. 518-528, Jan. 2010 
[9] X. Li et al, "A Controllable Untethered Vehicle Driven by Electrically Actuated Liquid Metal Droplets," IEEE Trans. Ind. Inform., Sep. 2018.

[10] J. Wu et al, "A wheeled robot driven by a liquid-metal droplet," Adv. Mater., vol.30, no.51, Oct. 2018.

[11] V. Sivan et al, "Liquid metals: liquid metal marbles (adv. funct. mater. 2/2013)," Adv. Funct. Mater., vol.23, no.2, pp. 137-137, Jan. 2013.

[12] H. Ota et al, "Highly deformable liquid-state heterojunction sensors," Nat. Commun., vol. 5, no. 5032, Sep. 2014.

[13] J. H. So et al, "Reversibly deformable and mechanically tunable fluidic antennas," Adv. Funct. Mater., vol. 19, no. 22, pp. 3632-3637, Nov. 2009.

[14] M. R. Khan et al, "A frequency shifting liquid metal antenna with pressure responsiveness," Appl. Phys. Lett., vol.99, no.1, 013501, Jul. 2011.

[15] J. Heikenfeld, P. Drzaic, J. S. Yeo and T. Koch, "Review paper: a critical review of the present and future prospects for electronic paper," J. Soc. Inf. Disp., vol.19, no.2, pp. 129-156, Jun. 2012.

[16] T. Krupenkin, S. Yang, and P. Mach, "Tunable liquid microlens," Appl. Phys. Lett., vol. 82, no. 3, pp. 316-318, Jan. 2003.

[17] S. Y. Tang et al, "Electrochemically induced actuation of liquid metal marbles." Nanoscale, vol. 5, pp. 5949-5957, Apr. 2013

[18] F. Li et al, "Magnetically-and electrically-controllable functional liquid metal droplets." Adv. Mater. Technol., Jan. 2019.

[19] J. Jeon, J. B. Lee, S. K. Chung and D. Kim, "On-demand magnetic manipulation of liquid metal in microfluidic channels for electrical switching applications," Lab Chip, vol.17, no.1, pp. 128-133, Nov. 2016.

[20] J. Shu et al, "Unconventional locomotion of liquid metal droplets driven by magnetic fields." Soft Matter, vol. 14, no. 35, pp. 7113-7118, Aug. 2018.

[21] J. Zhang, Y. Yao, L. Sheng, and J. Liu, "Self-fueled biomimetic liquid metal mollusk," Adv. Mater., vol. 27, no. 16, pp. 2648-2655, Mar. 2015.

[22] R. C. Gough et al, "Rapid electrocapillary deformation of liquid metal with reversible shape retention," Micro and Nano Syst Lett, vol.3, no. 4, Apr. 2015.

[23] X. Tang et al, "Photochemically induced motion of liquid metal marbles," Appl. Phys. Lett., vol. 103, no. 17, 174104, Oct. 2013.

[24] A. Zavabeti et al, "Ionic imbalance induced self-propulsion of liquid metals," Nat. Commun., vol. 7, 12402, Aug. 2016.

[25] J. Lee and C. J. Kim, "Surface-tension-driven microactuation based on continuous electrowetting," J. Microelectromech. Syst., vol. 9, no. 2, pp. 171-180, Jun. 2000.

[26] X. H. Yang, S. C. Tan, B. Yuan and J. Liu, "Alternating electric field actuated oscillating behavior of liquid metal and its application," Sci. China-Technol. Sci., vol. 59, no. 4, pp. 597-603, Apr. 2016.

[27] S. Y. Tang et al, "Liquid metal enabled pump," Proc. Natl. Acad. Sci. U. S. A., vol. 111, no. 9, pp. 3304-3309, Mar. 2014.

[28] J. Lee and C. J. Kim, 1999, Nov. Theory and modeling of continuous electrowetting microactuation. Presented at Proc. MEMS ASME IMECE '99 Vol. 1.

[29] J. Pellicer, V. Garcíamorales, and M. J. Hernández, "On the demonstration of the young-laplace equation in introductory physics courses," Phys. Educ., vol. 35, no. 2, pp. 144-168, Mar. 2000.

[30] J. Happel and H. Brenner, "Axisymmetrical Flow," in Low Reynolds number hydrodynamics, with special applications to particulate media, 1st ed, Netherlands: Martinus Nijhoff Publishers, 1983, pp. 127-129.C. C. Cheah, D. Q. Wang, and Y. C. Sun, "Region-reaching control of robots," IEEE Trans. Robot., vol. 23, no. 6, pp. 1260-1264, Dec. 2007.

[31] C. C. Cheah, D. Q. Wang, and Y. C. Sun, "Region-reaching control of robots," IEEE Trans. Robot., vol. 23, no. 6, pp. 1260-1264, Dec. 2007.

[32] H. Chen and D. Sun, "Moving groups of microparticles into array with a robot-tweezers manipulation system," IEEE Trans. Robot., vol.28, no. 5, pp. 1069-1080, Oct. 2012

[33] Y. Y. Yao and J. Liu, "A polarized liquid metal worm squeezing across a localized irregular gap," RSC Adv., vol.7, no.18, pp.11049-11056, Jan. 2017.

[34] A. Douglas, "Essentially follows Clarke," in Foundations of Analysis, 1st ed, New York: Appleton-Century-Crofts, 1971, pp. 284.

[35] A. D. Polyanin and A. V. Manzhirov, "Double and Triple Integrals," in Handbook of mathematics for engineers and scientists. 1st ed, Danvers: Chapman and Hall/CRC, 2007, pp.318.

[36] G. Beni, S. Hackwood, and J. L. Jackel, "Continuous electrowetting effect," Appl. Phys. Lett., vol. 40, no. 10, pp. 912-914, Aug. 1982

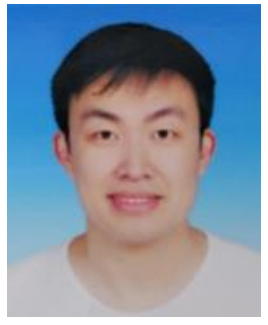

Jie Xie received the $\mathrm{BS}$ degree in mechatronic engineering from Soochow University, Soochow, China, in 2018. Since 2018, he begins to study toward his master degree in the Department of Precision Machinery and Precision Instrumentation of the University of Science and Technology of China. His research interests include liquid metal actuated robots and motion control.

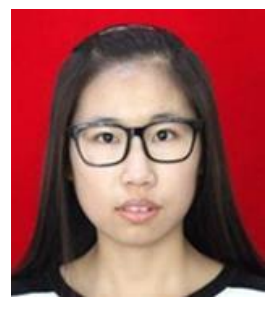

Fangxia Li received a BS degree in mechatronic engineering from Soochow University in 2017, and is a graduate student in the Department of Robotics at Soochow University. Her main research interests include smart material, liquid metal micro-robots and flexible robots.

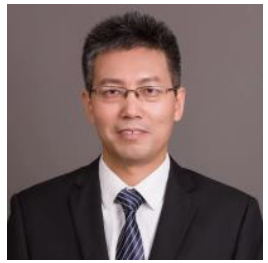

Shaolong Kuang received the bachelor's degree in mechanical manufacturing engineering from Anhui Polytechnic University, Wuhu, China, in 1996, the master's degree in mechanical manufacturing engineering from Southeast University, Nanjing, China, in 1999, and the Ph.D. degree in mechatronics engineering from Beihang University, Beijing, China, in 2013. He is currently an Associate Professor with Robotics and Microsystems Research Center, Soochow University, China. His research interests include medical robot and medical equipment design, and human-robot interaction.

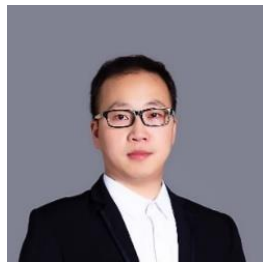

Hao Yang is currently an Associate Professor with the Robotics and Microsystems Center, College of Mechanical and Electrical Engineering, Soochow University, Suzhou, China. He received the B.E. degree in automatic control from the University of Science and Technology of China, Hefei, China, in 2010, and the Ph.D. degree in robotics and biomedical engineering from the University of Science and Technology of China, and City University of Hong Kong, Hong Kong, in 2015. From 2015 to 2016, he was a Post-Doctoral Researcher with the Department of Mechanical and Biomedical Engineering, City University of Hong Kong. His current research interests include robot-aided biomedical system, cell mechanism, micro/nano manipulation, and micro-robotic applications.

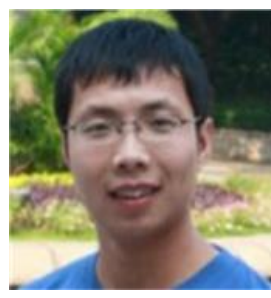

Xiangpeng $\mathbf{L i}$ is an associate professor in the Robotics and Microsystems Center, Soochow University, Suzhou, China. He received a B.Sc. in mechatronics and automation from Harbin Institute of Technology, Harbin, China, in 2006, and $\mathrm{PhD}$ in robotics and automation from both the University of Science and Technology of China and City University of Hong Kong in 2011. From 2011 to 2014, he was 
a post-doctoral researcher in the Department of Mechanical and Biomedical Engineering, City University of Hong Kong. His research interests include Micro-robots, soft robots and robotic assisted biomedical systems.

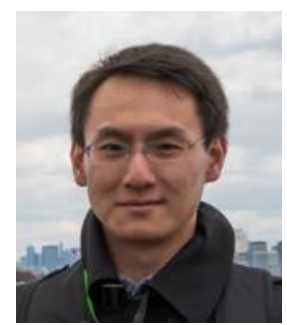

Shi-Yang Tang is a Vice- Chancellor's Postdoctoral Fellow at University of Wollongong, Australia. He received PhD in Microelectromechanical Systems from RMIT University, Australia in 2015. He joined Pennsylvania State University, USA, as a postdoctoral research fellow from 2015 to 2016. Later he joined the University of California, San Francisco (UCSF), USA, as a postdoctoral scholar from 2016 to 2017. His research interests include developing microfuidics platforms for biomedical studies and liquid metal enabled micro-/nanoscale platforms. $\mathrm{He}$ is the named author of over 45 papers in different peerreviewed journals (including PNAS, Advanced Materials, Advanced Functional Materials, Small, Analytical Chemistry, Lab on a Chip et al.) that have been cited $>1100$ times (H-index of 16) in just three years after his $\mathrm{PhD}$.

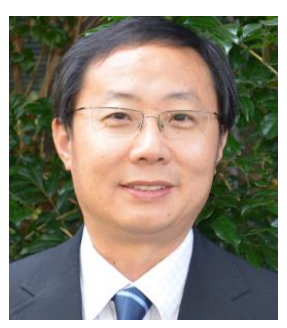

Weihua Li is a Senior Professor and Director of the Advanced Manufacturing Research Strength at the University of Wollongong. He completed his BEng (1992), MEng (1995) at University of Science and Technology of China, and $\mathrm{PhD}$ (2001) at Nanyang Technological University (NTU). He was with the School of Mechanical and Aerospace Engineering of NTU as a Research Fellow from 2001 to 2003, before he joined the University of Wollongong as a Lecturer. His research focuses on smart materials and their applications, microfluidics, lab on a chip, rheology, and intelligent mechatronics. He serves as editor or editorial board member for more than 11 international journals. He has published more than 350 journal and conference papers. He is a recipient of Fellow of the Engineers Australia, the Fellow of Institute of Physics (UK), Australian Endeavour Research Fellowship, JSPS Invitation Fellowship, Vice-chancellor's Award for Interdisciplinary Research Excellence, and numerous Best Paper Awards.

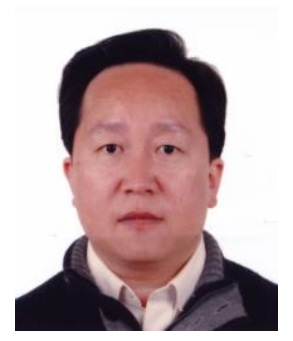

Shiwu Zhang (M'13) received the BS degree (1997) in mechanical and electrical engineering from University of Science and Technology of China (USTC), and the $\mathrm{PhD}$ degree (2003) in precision instrumentation and precision machinery from USTC, Hefei, China. He is currently a professor in the Department of Precision Machinery and Precision Instrumentation, USTC. He became a Member (M) of IEEE in 2012. He is the named author of over 100 papers in different peer-reviewed journals and conferences. His current research interests include precision instrumentation, smart materials and their applications in bio-inspired robots, and soft robots. 